\title{
Produção de vídeos como material didático de apoio para aprendizagem em saúde da mulher: relato de experiência
}

\section{Video production as supporting educational material for women's health learning: experience report}

\author{
${ }^{1}$ Julio Cesar Soares Aragão jaragaum@gmail.com \\ ${ }^{1}$ Gabriela de Azevedo Rosestolato Soares \\ ${ }^{1}$ Ian Xavier Paschoeto dos Santos \\ ${ }^{1}$ Marina Coelho de Souza \\ ${ }^{1}$ Renata Sydio de Souza
}

\section{RESUMO}

Na sala de aula convencional, os alunos têm o contato com o conteúdo educacional apenas através das aulas centralizadas no professor e, posteriormente, com as lições de casa, evidenciando que os acadêmicos de medicina expressam uma forte apreciação pela metodologia ativa "Flipped Classroom”, que inclui diversas atividades preparatórias para o momento de sua aplicação em aula. Em meio às inovaç̃os tecnológicas e à inserção do indivíduo no mundo digital, essas atividades, facilitadas por ferramentas online, promovem aumento da interatividade, da absorção do conteúdo, um menor custo, uma excelente qualidade de ensino e uma maior autonomia do educando. Busca-se que o estudante de medicina consolide o conhecimento de saúde através do material didático, o qual será produzido, beneficiando futuramente o meio médico com profissionais humanizados e bem informados. O trabalho conta com a produção de vídeo aulas abordando o tema de saúde da mulher de acordo com o ciclo básico no Curso de Medicina. Esses vídeos foram produzidos pelos alunos do curso, dando atenção aos conteúdos de embriologia do aparelho reprodutor feminino e fisiologia do ciclo menstrual. O projeto inclui a criação e o planejamento, que definem o assunto abordado no vídeo, e a finalidade do projeto, que visou aprimorar o conhecimento do aluno, facilitando o processo de aprendizagem. Posteriormente, houve a criação do roteiro, que consiste em um resumo do conteúdo, baseado nos livros didáticos recomendados pelo corpo discente do Curso de Medicina do Centro Universitário de Volta Redonda, dos quais também foram retiradas imagens que pudessem auxiliar na produção. Tão importante quanto, foi a pré-produção, que define a função de cada um que participará da produção do material, como por exemplo, para quem estará designado o cargo de produtor, editor, narrador, capturador de vídeo e outras atividades que possam vir a serem necessárias. Em seguida, houve a etapa de edição e finalização, que contou com a organização do material e a junção das montagens em vídeos de cerca de dez minutos. O software utilizado para edição pode ser encontrado para download gratuito. Ao final da produção da vídeo-aula, o projeto foi publicado no YouTube.

Palavras-chave: Educação médica. Tecnologia da informação. Saúde da mulher.

\section{ABSTRACT}

In conventional classroom, students have contact with educational content only through teacher-centered lessons and, later, through homework, demonstrating that medical students express a strong appreciation for the active Flipped Classroom methodology, which includes several preparatory activities for its application in class. In the technological's midst innovations and the individual's insert in the digital world, these activities, facilitated by online tools, promote increased interactivity, content absorption, lower cost, excellent teaching quality and greater student autonomy. It is intended that the medical student consolidate the health's knowledge through the teaching material, which will be produced, benefiting the medical environment in the future with humanized and well-informed professionals. The work is about video lessons production addressing the topic of women's health according to the basic cycle in the Medicine Course.

1 Centro Universitário de Volta Redonda, UniFOA 
Those videos were produced by the students, focusing on the female reproductive tract's embryology and the menstrual cycle. The project includes the creation and planning, which define the subject addressed in the video, and the project's purpose, which aimed to improve student knowledge, upgrading the learning process. Subsequently, the script was created, which consists in a content's summary, based on the textbooks recommended by the student body of the Centro Universitário de Volta Redonda's medical school, from which were also taken images that could aid in the production. As important as it, was the pre-production, which defines the role of each one who will participate in the material production, for example, for whom will be designated the position of producer, publisher, narrator, video grabber and other activities that may come needed. Then, there was the editing and finishing stage, which included the material's organization and the montages' joining in videos, that have around ten minutes. The software used for editing can be found for download at nocharge. At the end of the production of the video lesson, the project was published on YouTube.

Keywords: Medical education. Information technology. Women's health.

\section{INTRODUÇÃO}

Atividades voltadas para ao ensino e a educação são tão antigas quanto o próprio homem e podem ser encontradas desde os ensinamentos de Platão (Atenas Clássica), até as instituições católicas implementadas pelos jesuítas no Brasil. A forma de sua implantação é discutida em várias épocas, visando aumentar sua eficiência ou atender um ideal político, como a Reforma Pombalina do poder imperial (FARIAS; MARTIN; CRISTO, 2015).

A chamada educação bancária, por exemplo, foi denunciada por Paulo Freire, que procurava empregar uma pedagogia libertadora, problematizadora e conscientizadora. A ideia bancária consiste em o educador transmitir e depositar a palavra no educando que deve escutar e preencher seu espaço vazio reservado ao aprendizado. Nota-se a acusação de um sistema de opressão, que necessita de disciplina, obediência e implementação das regras, visualizado em muitas salas de aula. Por outro lado, a conscientização transforma o educando em sujeito e o educador em problematizador e libertador, o qual trabalha com perguntas, integrando todos no debate. Dessa forma, inicia-se a ideia da implementação de metodologias ativas no ambiente acadêmico (BRIGHENTE; MESQUIDA, 2016).

Tais metodologias ativas de ensino são construídas a partir das discussões que procuram reestabelecer as relações entre o educando e o educador, o que garantem os objetivos de libertação dos antigos métodos de educação. Todavia, para que esse processo se concretize, a fim de garantir a autonomia, desenvolvimento do conhecimento e a reflexão das ideias pelo educando, faz-se necessária a multiplicidade de metodologias e a aplicação efetiva pelo educador. Desse modo, é fundamental que a instituição estabeleça o diálogo e métodos de integração na relação educador-educando, assim como a autonomia do aluno, mesmo que necessite de sua readequação. O professor deve facilitar a transmissão e o aluno deve desenvolver o conhecimento adquirido dentro do seu espaço e de suas relações, atribuindo dinâmica ao aprendizado (FARIAS; MARTIN; CRISTO, 2015).

A interatividade estudante-professor presente na sala de aula é fundamental para o aprendizado, impondo um desafio a ser vencido pela criatividade do educador tanto na educação à distância, assim como na presencial. Portanto, a qualidade do material didático produzido irá influenciar na compreensão do aluno, já que quanto mais diversificado e criativo este for, de maior qualidade será (PASSOS; BARBOSA, 2012).

A produção de material que cative a audiência do usuário demanda planejamento do conteúdo e do formato de suas aulas, assim como a postura do professor, cenário, equipamentos necessários e uma boa edição. É necessário, também, que utilize um bom hospedeiro e que haja uma boa divulgação. Além disso, é essencial que seja disponibilizada uma apostila de estudos online para o aluno, de forma que a aula seja melhor compreendida, podendo conter exercícios, resumos ou referências. Animações e conferências online também são úteis para que haja uma maior interação entre as duas partes (PASSOS; BARBOSA, 2012). 
Em complemento às aulas, a produção de vídeos também deve seguir uma série de critérios para sua efetivação, como: criação e planejamento; roteiro; pré-produção; edição e finalização. O desafio educacional não é somente democratizar as Tecnologias Digitais da Informação e Comunicação, mas também explorá-las no processo de aprendizagem médica. As metodologias ativas, como as mediadas por materiais eletrônicos, são interessantes para a nova geração, que está amplamente integrada no mundo tecnológico, além de atingirem um maior número de indivíduos (SATO, 2015)vinculada à linha Tecnologias da Informação e da Comunicação na Educação Básica, do Programa de Pós-Graduação-Mestrado em Docência para Educação Básica da Faculdade de Ciências da Universidade Estadual Paulista- UNESP, surgiu diante de indagações enquanto docente dos anos iniciais do Ensino Fundamental, no que tange a questão do uso das Tecnologias Digitais da Informação e Comunicação (TDIC.

Em uma sala de aula convencional, os alunos têm o contato com o conteúdo educacional apenas através das aulas centralizadas no professor e, posteriormente, com as lições de casa. Para o modelo "Flipped Classroom” (ou sala de aula invertida, em português), ou sala de aula invertida, é possível que haja uma exposição do conteúdo aos alunos antes das aulas, por meio de leituras, vídeos ou outros materiais eletrônicos ministrados de acordo com a escolha do professor, potencializando o ensino (RAMNANAN; POUND, 2017)"container-

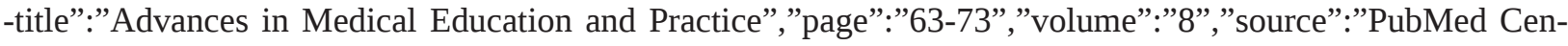
tral","abstract”:”'The flipped classroom (FC.

Estudantes de medicina expressam uma forte apreciação com salas de aula invertidas, que incluem diversas atividades preparatórias para o momento de sua aplicação em aula. Em meio às inovações tecnológicas e à inserção precoce do indivíduo no mundo digital, essas atividades, quando facilitadas por ferramentas online, aumentam a interatividade e a absorção do conteúdo pelo aluno, promovem um menor custo, uma excelente qualidade de ensino e, principalmente, uma maior autonomia do educando (RAMNANAN; POUND, 2017)"container-title”:”Advances in Medical Education and Practice”,"page”:"63-73”,"volume":"8”,"source":"PubMed Central”,"’abstract”:”The flipped classroom (FC.

Segundo VALENTE (2017), o ensino superior, atualmente, tem enfrentado grandes desafios. As salas de aulas estão cada vez mais vazias e, quando o aluno está presente, ele está fazendo algo que não faz parte do acompanhamento à aula. Outro desafio enfrentado é a incapacidade de atender a grande demanda de alunos que querem ingressar no ensino superior. Sendo assim, é fundamental que o método de ensino tradicional seja modificado de forma que procure amenizar essas adversidades.

As Diretrizes Curriculares Nacionais para Cursos de Medicina (BRASIL, 2014) são claras em preconizar um ensino "centrado no aluno como sujeito da aprendizagem e apoiado no professor como facilitador e mediador do processo". A leitura deste documento deixa clara a intencionalidade dos legisladores em introduzir inovações no ensino médico com a utilização de tecnologias diferenciadas que visam propiciar uma nova realidade educacional. Esse novo sistema, então, permite que seja utilizado o método da produção de vídeo-aulas, em razão de aprofundarem o conhecimento do educando, além de promoverem uma maior autonomia desse, que, antes, dependia completamente do educador para desenvolver o seu processo de aprendizagem. Outra contribuição seria a democratização da informação, já que permite que a abordagem de qualquer temática ocorra de maneira mais expansiva, visto que, atualmente, a grande maioria da população possui fácil acesso à Internet. Ademais, as tecnologias da informação e comunicação, em especial o vídeo, podem vir a significar novos métodos de aprendizagem e ensino para que tanto os alunos quanto os professores possam utilizá-las como ferramentas de reflexão e/ou recurso didático-pedagógico (LAMPERT; REGO; ARAÚJO, 2004).

Segundo LIMA (2017), o início da utilização de metodologias ativas na educação é advindo do movimento escola novista, caracterizado pelo questionamento à passividade na qual o estudante estava condenado diante da escola tradicional. O processo de ensino e aprendizagem, contaminado por essas técnicas tradicionais, tem se restringido, diversas vezes, à reprodução do conhecimento, no qual o educador transmite os conteúdos e ao educando é designada a tarefa de reter e repetir os mesmos, tornando-se um mero expectador do seu processo 
de aprendizagem, sem a crítica e a reflexão necessárias para que haja um ensino concreto. As novas tecnologias permitem aos educandos o poder de produzir uma capacidade crítica e reflexiva sobre o que estão aprendendo e promover um maior comprometimento do aluno no processo educacional, além de uma vinculação dos assuntos abordados à realidade vivida, um maior desenvolvimento do raciocínio e a colaboração e cooperação entre participantes. Existem dois desafios diante dessa aquisição de novas metodologias: tornar acessíveis as tecnologias voltadas à aprendizagem ativa e considerar uma abordagem crítica e interdisciplinar na seleção dos conteúdos compartilhados.

Na saúde, as dimensões subjetiva e social do fenômeno saúde-doença têm sido, durante anos, descontextualizadas do método de aprendizagem, já que "na maioria das escolas médicas ainda encontramos uma estrutura curricular atrelada ao modelo flexneriano, caracterizado pela divisão em disciplinas básicas e clínicas, destacando-se a importância das especializações e dos departamentos e enfatizando-se os aspectos puramente biológicos” (CARABETTA, 2016). Sendo assim, o perfil do profissional usualmente formado é questionado, principalmente diante da preocupação com a tendência à especialização precoce.

Diante da grande ênfase na formação em ciências básicas nos primeiros anos, da organização minuciosa da assistência médica em várias especialidades individuais, da valorização do ensino centrado no ambiente hospitalar e com foco na atenção curativa e individual, o ensino nessa área é distanciado do serviço e das necessidades reais do Sistema Único de Saúde (SUS), tendo formado, em grande parte, profissionais não afeitos aos princípios da humanização e interdisciplinaridade, competências essas fundamentais para a sua formação (MITRE et al., 2008). Para esse motivo, é também introduzida a questão de revolucionar os métodos de ensino, dado que a utilização de metodologias ativas, além de propiciar uma educação abrangente e de alta qualidade, relaciona as diversas disciplinas do campo da saúde sem a necessidade da compartimentalização do ensino médico (LAMPERT, 2009).

Para propiciar interdisciplinaridade e humanização, há um movimento voltado à produção de mudanças tanto em relação às metodologias ativas de ensino, quanto à promoção de uma educação possivelmente transformadora. Dessa forma, essas metodologias vêm sendo utilizadas na formação e capacitação de profissionais da área de saúde, de maneira estratégica para a integração de saberes e para a promoção de uma atitude crítica e reflexiva sobre a prática (LIMA, 2017).

Universidades como MIT e Harvard adotaram a estratégia da "sala de aula invertida”, implantada em algumas disciplinas. Desse modo, há a inovação dos métodos de ensino nessas escolas, procurando adequá-los para que possam explorar os avanços das tecnologias educacionais, bem como minimizar a evasão e o nível de reprovação entre os estudantes. Harvard, por exemplo, adotou um método em que os alunos provêm de um material de apoio enviado antes das aulas, de maneira que possam estar preparados antes da reunião com o professor. Com base nesse material previamente disponibilizado, responderão a um questionário que, posteriormente, será discutido em grupos de três a quatro pessoas. Ao utilizar essa estratégia, são apresentados ganhos significativos na compreensão conceitual por parte dos educandos, bem como esses adquirem habilidades para resolver problemas de maneira mais fundamentada do que em comparação aos adquiridos nas aulas tradicionais (VALENTE; MORAN; BACICH, 2017).

Fica evidente a importância e a implantação diferenciada de modelos para a inovação das escolas médicas atuais. Em complemento, RAMNANAN e POUND (2017)"container-title":"Advances in Medical Education

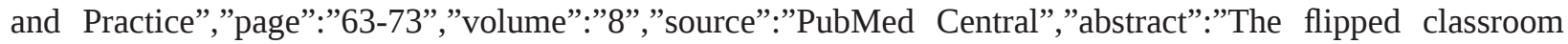
(FC indicam que os padrões analisados para a acreditação do curso de medicina exigem, formalmente, a inclusão de metodologias ativas, uma vez que elas permitem a aprendizagem a longo prazo do aluno, o qual se tornará um médico estabelecido na modernidade. Os resultados de seus estudos demonstraram uma reação positiva dos estudantes de medicina ao modelo de ensino ativo e grande apreciação aos recursos de introdução de materiais eletrônicos antes da ocorrência da aula, tais como as vídeo-aulas. 
De acordo com Sousa, Moita e Carvalho (2011)11,27]]\}\},"suppress-author":true\}],"schema”:"https://github.com/citation-style-language/schema/raw/master/csl-citation.json”\} , a utilização de vídeo aulas englobando temas específicos, no caso, envolvendo a saúde da mulher, tema constantemente marginalizado e necessitado de investimento, permite estender as habilidades cognitivas, a memória, o compartilhamento de saberes e materiais, e a busca por informações disponíveis no meio eletrônico. Dessa forma, o estudante de medicina ampliará seu material de estudo e terá maior motivação e aprendizagem, já que ferramentas e mídias digitais oferecem instrumentos capazes de renovar as situações de interação, expressão, criação, comunicação, informação, e colaboração, tornando-a muito diferente daquela tradicionalmente fundamentada na escrita e nos meios impressos (SOUSA; MOITA; CARVALHO, 2011)11,27]]\}\}\}],”schema”:”https://github.com/citation-style-language/sche$\mathrm{ma} /$ raw/master/csl-citation.json”\} .

Na dimensão metacognitiva, espera-se que os alunos que conhecem diferentes estratégias de aprendizagem provavelmente irão utilizá-las para pensar e resolver problemas. Estudantes que estejam conscientes dos seus pontos fortes e limitações pessoais podem ajustar a aprendizagem e contribuir para estratégias de aprendizagem mais favoráveis às suas necessidades (FARIAS; MARTIN; CRISTO, 2015, p. 149).

A proposta de abordar a saúde da mulher também reivindica a sua reduzida atenção na história política de saúde no Brasil, a qual procurou, por muitos anos, otimizar apenas os parâmetros materno-infantil. "A criação do PAISM, em 1984, foi um marco e resultado das reivindicações dos movimentos de mulheres, que exigiam principalmente que o atendimento no sistema de saúde levasse em conta a integralidade da pessoa: a mulher passou a ser vista não apenas como a mulher que engravidava e tinha filhos, mas também a que tinha direitos”. Todavia, a implementação não foi efetiva a ponto de apresentar resultados significativos, sendo frequentes situações em que a assistência fica aquém dos parâmetros preconizados como ideais. Por esse motivo, faz-se necessária a ampliação do conceito de saúde da mulher, partindo, principalmente, da educação daqueles que propagam a informação e praticam as ações em saúde diariamente: os médicos (BOYACIYAN; CAMANO, 2006; RATTNER, 2009).

O objetivo desse estudo é relatar a experiência de desenvolver material didático, produzindo vídeo-aulas que abordam disciplinas básicas que têm por objeto de estudo a morfofuncionalidade do sistema genital feminino, de forma a ampliar as opções de fomento ao processo de ensino e aprendizagem, restrito, em alguns casos, à reprodução do conhecimento transmitido pelo educador.

\section{MÉTODO}

Optamos pelo relato de experiência por se tratar de uma forma simples e direta de compartilhamento de vivências que permite descrever as ações de ensino, assim como refletir sobre as atividades desenvolvidas (ARAGÃO, 2013). Essa categoria de produção científica parte de um conjunto de dados contextuais que ensejam uma análise ampla sobre uma determinada prática a partir de uma perspectiva de inovação ou aprimoramento contínuo de processos de trabalho (BIANCOLINO et al., 2011), constituindo não apenas um momento de divulgação de práticas, mas também um momento de reflexão dialógica entre o conhecimento teórico e as experiências advindas da prática, em um processo no qual a contraposição entre a teoria e a prática gera um ciclo de aproximações mútuas entre os dois campos, permitindo adaptações da realidade e questionamentos que possam reiniciar todo o processo continuamente.

No campo do ensino, tais processos são vivenciados não somente pelos alunos, mas principalmente pelos professores que se disponibilizem a "repensar o pensado" (FREIRE, 2001, p. 259) e estabelecer novas práxis pedagógicas que o aproximem do aluno, instiguem a curiosidade e busquem novas realidades que ampliem a construção do conhecimento tanto discente quanto docente. 


\section{RELATO DA EXPERIÊNCIA}

Algumas etapas foram fundamentais no processo de produção. Dentre elas estão a criação e o planejamento, que definem o assunto abordado no vídeo, de acordo com os citados acima, e a finalidade do projeto, que visou aprimorar o conhecimento do aluno, facilitando o processo de aprendizagem. Posteriormente, houve a criação do roteiro, que consiste em um resumo do conteúdo, baseado nos livros didáticos recomendados pelo corpo docente do Curso de Medicina do Centro Universitário de Volta Redonda (MONTENEGRO; REZENDE, 2018; ZUGAIB, 2015).

Após a pré-produção, na qual ocorre a definição da função de cada um na produção do material (por exemplo, para quem estará designado o cargo de produtor, editor, narrador, capturador de vídeo e outras atividades que possam vir a serem necessárias), foi seguida, pela etapa de edição e finalização, que contou com a organização do material e a junção das montagens em dois vídeos de aproximadamente cinco minutos. O software utilizado para edição foi o iMovie, que pode ser encontrado para downloadgratuiti de dispositivos de telefonia móvel (HANDLER et al., 2001). No entanto, é possível a utilização de outros aplicativos, de acordo com a preferência e experiência dos participantes.

Ao final da produção, as vídeo-aulas foram publicadas no YouTube, de forma a garantir a acessibilidade a todo o público a quem possa interessar o assunto discutido.

\section{CONSIDERAÇÕES FINAIS}

O estudante, ao produzir material didático em forma de vídeo-aulas que focassem em assuntos tratados durante o Curso, permitiu que o conteúdo fosse fixado de maneira mais efetiva, pois desenvolve a capacidade de ensinar. Além disso, a utilização de vídeo-aulas incentiva a autonomia, a disciplina e a reflexão daquele que assiste, libertando-o do modelo tradicional, no qual o educador transmite o conhecimento e o educando apenas o repete.

Nesse contexto, novas metodologias devem ser estudadas e aprimoradas, pois nota-se a melhora do aproveitamento do aluno na administração e compreensão de seus estudos. Sendo assim, a utilização dessas tecnologias de ensino em um trabalho relacionado à saúde da mulher é de extrema relevância, uma vez que o conhecimento adquirido pelos estudantes sobre o tema no período analisado será, então, aplicado futuramente em sua formação acadêmica e profissional.

Busca-se, assim, que o estudante de medicina consolide o conhecimento de saúde da mulher através do material didático, o qual foi produzido, beneficiando futuramente o meio médico com profissionais humanizados e bem informados. Com os objetivos atingidos de forma satisfatória, os presentes métodos podem ser estendidos a outras áreas de conhecimentos das ciências da saúde. 


\section{REFERÊNCIAS}

ARAGÃO, J. Introdução aos estudos quantitativos utilizados em pesquisas científicas. Revista Práxis, v. 3, n. 6, 10 fev. 2013.

BIANCOLINO, C. A. et al. PROTOCOLO PARA ELABORAÇÃO DE RELATOS DE PRODUÇÃO TÉCNICA. jul. 2011.

BOYACIYAN, K.; CAMANO, L. O perfil dos médicos denunciados que exercem ginecologia e obstetrícia no estado de São Paulo. Rev Assoc Med Bras, p. 4, 2006.

BRASIL. RESOLUÇÃO No 3, DE 20 DE JUNHO DE 2014. Diretrizes Curriculares Nacionais do Curso de Graduação em Medicina. . 2014, p. 47.

BRIGHENTE, M. F.; MESQUIDA, P. Paulo Freire: da denúncia da educação bancária ao anúncio de uma pedagogia libertadora. Pro-Posições, v. 27, n. 1, p. 155-177, abr. 2016.

CARABETTA, V. J. Metodologia ativa na educação médica. Revista de Medicina, v. 95, n. 3, p. 113-121, 15 dez. 2016.

FARIAS, P. A. M. DE; MARTIN, A. L. DE A. R.; CRISTO, C. S. Aprendizagem Ativa na Educação em Saúde: Percurso Histórico e Aplicações. Revista Brasileira de Educação Médica, v. 39, n. 1, p. 143-150, mar. 2015.

FREIRE, P. Carta de Paulo Freire aos professores. Estudos Avançados, v. 15, n. 42, p. 259-268, ago. 2001.

HANDLER, M. et al. iMovie and Educators: the Right Partnership for Making Digital Movies.. In: SOCIETY FOR INFORMATION TECHNOLOGY \& TEACHER EDUCATION INTERNATIONAL CONFERENCE. Association for the Advancement of Computing in Education (AACE), 2001Disponível em: <https://www. learntechlib.org/primary/p/16960/>. Acesso em: 6 nov. 2019

LAMPERT, J. Tendências de mudanças na formação médica no Brasil. 2. ed. São Paulo: Hucitec/Associação Brasileira de Educação Médica, 2009. v. 1

LAMPERT, J. B.; REGO, S.; ARAÚJO, J. G. C. Educação médica em transformação: instrumentos para a construção de novas realidades. [s.l.] Editora Hucitec, 2004.

LIMA, V. V. Espiral construtivista: uma metodologia ativa de ensino-aprendizagem. Interface - Comunicação, Saúde, Educação, v. 21, n. 61, p. 421-434, jun. 2017.

MITRE, S. M. et al. Metodologias ativas de ensino-aprendizagem na formação profissional em saúde: debates atuais. Ciência \& Saúde Coletiva, v. 13, n. suppl 2, p. 2133-2144, dez. 2008.

MONTENEGRO, C. A. B.; REZENDE, J. DE. Rezende obstetrícia fundamental. 14. ed. [s.l.] Grupo Gen Guanabara Koogan, 2018.

PASSOS, F. V.; BARBOSA, T. R. C. G. Produção de material didático. Viçosa: Coordenadoria de Educação Aberta e a Distância, 2012.

RAMNANAN, C. J.; POUND, L. D. Advances in medical education and practice: student perceptions of the flipped classroom. Advances in Medical Education and Practice, v. 8, p. 63-73, 13 jan. 2017.

RATTNER, D. Saúde da Mulher. In: DALLER, V. L. O.; MOREIRA, E. DE M. A. (Eds.). . Cooperativismo de gênero. Brasília/DF: Ministério da Agricultura, Pecuária e Abastecimento, Secretaria de Desenvolvimento Agropecuário e Cooperativismo, 2009. 
SATO, M. A. V. [UNESP. Tecnologias Digitais da Informação e Comunicação: explorando as possibilidades pedagógicas da produção de vídeos. 17 dez. 2015.

SOUSA, R. P. DE; MOITA, F. DA M. C. DA S. C.; CARVALHO, A. B. G. Tecnologias digitais na educação. [s.l.] EDUEPB, 2011.

VALENTE, J. A.; MORAN, J.; BACICH, L. A sala de aula invertida e a possibilidade do ensino personalizado: uma experiência com a graduação em midialogia. In: Metodologias ativas para uma educação inovadora. [s.l.] Penso, 2017. p. 19.

ZUGAIB, M. Zugaib Obstetrícia. Edição: 3a ed. [s.l.] Manole, 2015. 